\title{
Development of Antimicrobial Membranes via the Surface Tethering of Chitosan
}

\author{
R. Nigmatullin, ${ }^{1}$ V. Konovalova, ${ }^{2}$ G. Pobigay ${ }^{2}$ \\ ${ }^{1}$ School of Science and Technology, Nottingham Trent University, Clifton Lane, Nottingham NG11 8NS, \\ United Kingdom \\ ${ }^{2}$ Department of Chemistry, National University of Kyiv-Mohyla Academy, 2 Skovoroda Street, Kiev 04070, \\ Ukraine
}

DOI 10.1002/app.29135

\begin{abstract}
To render the surface of ultrafiltration membranes biocidal, cellulose membranes were modified with chitosan, a naturally occurring polycationic biocide. Through the use of chitosans of different molecular weights and membranes with different pore sizes, the alteration of the morphological structure of tethered chitosan layers was achieved. The importance of such structural differences in the antimicrobial activity of the prepared membranes against gram-positive Staphylococcus aureus and gram-negative Escherichia coli was studied. The antimicrobial efficiency improved with the use of chitosans
\end{abstract}

with higher molecular weights and membranes with smaller pore sizes. This suggested that the surface location of the grafted chitosan chains was more preferential for a higher antimicrobial activity of the surface. Membranes modified with chitosan showed higher antimicrobial efficiency against gram-positive $S$. aureus than against gramnegative E. coli.

Key words: biological applications of polymers; biopolymers; membranes; modification; surfaces

\section{INTRODUCTION}

Membrane biofouling is a widely recognized operational problem of water-treatment plants. ${ }^{1-7}$ Autopsical studies of reverse-osmosis and nanofiltration membranes fouled during the treatment of different types of surface water ${ }^{2,8}$ and wastewaters ${ }^{9,10}$ have shown that more than $50 \%$ (in most cases $80-90 \%$ ) of the dry weight of the fouling layers is biological in origin. The surface density of viable cells in the fouling layers ranges from $10^{6}$ to $10^{8} \mathrm{CFU} / \mathrm{cm}^{2}$. Thus, fouling layers are actually biofilms with a structure and cellular physiological behavior enabling microorganisms to survive under relatively harsh conditions of hydrodynamic shear and, in the case of surface water, a low-nutrient environment.

The main concern relevant to membrane fouling is increased operating costs due to a gradual decline in permeate water flux, the necessity of higher operating pressures and frequent chemical cleanings, and shortened membrane life. Moreover, biofouling can

Correspondence to: R. Nigmatullin (rinat.nigmatullin@ntu. ac.uk) and V. Konovalova (vita@ukma.kiev.ua). also affect the quality of treated water as a result of a higher level of concentration polarization and possible secondary contamination with metabolic products. ${ }^{11}$

Unlike other types of fouling (scaling and deposition of colloid particles), biofouling is barely controllable via the employment of different pretreatment stages (chlorination, biological exhaustion of nutrient species, etc.). In large- to medium-scale technical systems, such measures neither lead to sterility nor maintain it over a long period of time. Even a diminutive survival rate eventually will result in colonization of the membrane surface with microorganisms due to the growth of colonies initiated by cells that survive. ${ }^{3}$

Although it is generally accepted that biofilm formation is poorly understood, cell attachment to the membrane surface and subsequent stabilization of the cell association with the surface are considered crucial stages in biofilm formation. Viable cells produce adhesive extracellular polymeric substances that improve microorganism binding to the membrane surface and thereby enhance the survival and robustness of the biofilm microorganisms. Therefore, an alternative approach to controlling membrane biofouling can be based on interfering with these critical events of biofilm formation. Membrane biofouling potential could be crucially reduced if the surface properties of the membrane prevent cell 
adhesion or if the attached cells are not able to survive on the membrane surface. In the latter case, the membrane surface has to be biocidal or at least bacteriostatic.

The propensity of a solid surface to attach cellular particles depends on both the surface characteristics (surface charge, roughness, etc.) and environmental conditions (ionic strength, $\mathrm{pH}$, etc.). A number of studies have demonstrated the feasibility of reducing biomaterial adhesion to a membrane surface via the variation of the membrane surface properties. ${ }^{12-15}$ However, all such research has been carried out with a model single foulant. The feasibility of this approach of biofouling control for the treatment of natural waters with their complex biota and biomolecule stock is still to be explored.

In recent years, self-sterilizing surfaces have attracted growing interest. Products with an added antimicrobial treatment are finding excellent acceptance by the medical community. They include surgical drapes, instrument wraps, and surgical packs that reduce the risk of postoperative infection ${ }^{16,17}$ and tooth fillings. ${ }^{18}$ Microbiological evaluations have been carried out on a variety of textile materials ${ }^{19}$ and food packaging ${ }^{20}$ treated with antimicrobial agents. They have been treated against a broad spectrum of microorganisms, including odor-causing bacteria as well as bacteria and fungi, which cause rot and mildew. Most such materials are based on compositions that release biocidal molecules or ions. However, the application of polymer biocides has opened new frontiers in the development of nonleaching antibacterial surfaces. ${ }^{21,22}$ Recently, attempts have been made to render membrane surfaces antimicrobial by graft copolymerization and interfacial polycondensation of amine-containing polymers, which are potentially antimicrobial agents. ${ }^{23,24}$ It has been shown that membranes modified with layers of quaternary 2-dimethylaminoethylmethacrylate and crosslinked poly(ethylene imine) drastically suppress the viability of Escherichia coli cells and potentially are less prone to biofouling.

In this study, chitosan, the antimicrobial properties of which are well documented, ${ }^{25}$ was tethered to the surfaces of cellulose membranes to provide them biocidal activity and thereby lower the membrane biofouling potential. Chitosan samples of two molecular weights were used to modify cellulose membranes of different pore sizes with molecular weight cutoffs (MWCOs) ranging from 5 to $100 \mathrm{kDa}$. The membrane bactericidal activity was tested against E. coli and Staphylococcus aureus strains. The influence of the modification conditions, molecular weight of the chitosan, and morphological structure of the membranes on the antimicrobial properties of the prepared membranes was studied.

\section{EXPERIMENTAL}

\section{Materials}

Chitosan samples with molecular weights of approximately 400 and $150 \mathrm{kDa}$ were purchased from Fluka (Loughborough, UK) and used without any purification. The degree of deacetylation of both materials was about $85 \%$. Membrane modification was carried out with chitosan solutions prepared by the dissolution of chitosan in a $1 \mathrm{wt} \%$ acetic acid solution.

Poly(ethylene glycol) (PEG), sodium periodate, and sodium borohydride were purchased from Sigma-Aldrich (Loughborough, UK). All the chemicals were analytical-grade and were used without further purification.

Cellulose membranes C005F, C010F, C030FM, and $\mathrm{C} 100 \mathrm{~F}$ were purchased from Microdyn Nadir (Wiesbaden, Germany). According to the manufacturer's specifications, these membranes had MWCOs of 5000, 10,000, 30,000, and 100,000, respectively. The membranes were asymmetric with a thin active layer of regenerated cellulose supported by a porous hydrophobic polymer film.

\section{Modification of the cellulose membrane}

Scheme 1 illustrates the overall modification procedure for binding chitosan to a cellulose surface. Modification of the cellulose membrane was based on oxidative cellulose activation via an introduction of dialdehyde units into cellulose macromolecular chains by periodate oxidation. A subsequent reaction of the oxidized cellulose membrane with aqueous chitosan solutions resulted in covalent chitosan binding through the formation of imine groups (Schiff base), which subsequently were reduced to form more stable carbon-nitrogen bonds.

The cellulose membrane samples were oxidized by incubation in $0.1 \mathrm{M}$ sodium periodate for $1 \mathrm{~h}$ at $55^{\circ} \mathrm{C}$. After oxidation, the membranes were rinsed with deionized water produced by the Millipore Milli-Q Plus system (Watford, UK) to remove residues of the oxidizing agent. Activated membrane samples were transformed into chitosan solutions of different concentrations to bind chitosan to the membrane surface. Preliminary studies demonstrated that if the contact time of activated cellulose membranes with chitosan solutions is less than $10 \mathrm{~h}$, only low degrees of modification are achievable at any chitosan solution concentration. Therefore, in this study, all modified membranes were prepared through the reaction of oxidized cellulose samples with chitosan for $20 \mathrm{~h}$ at room temperature. The chitosan concentration was varied from 0.1 to 2 wt \%.

Modified membranes were rinsed with deionized water produced by the Millipore Milli-Q Plus system and stabilized by reduction in a $0.1 \mathrm{M} \mathrm{NaBH}$ 


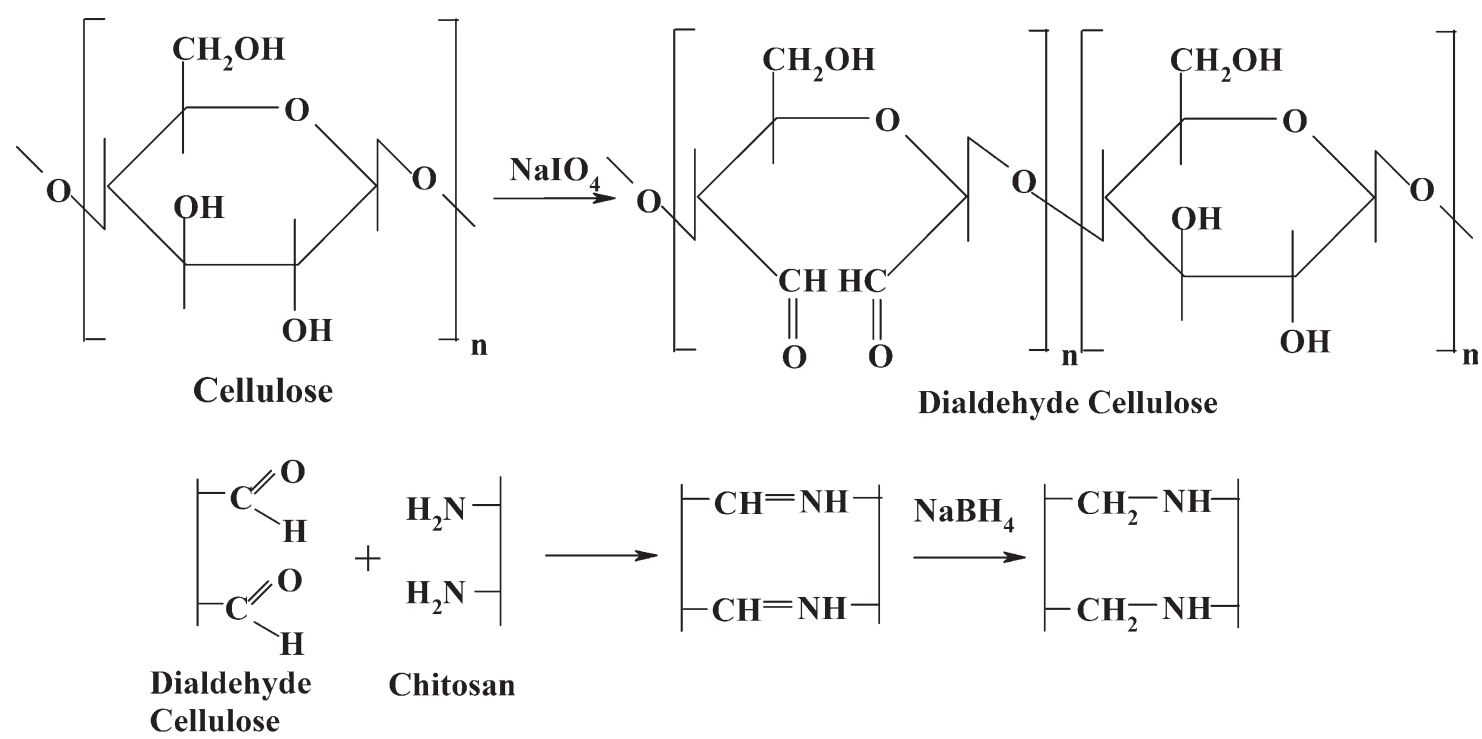

Scheme 1 Chemical transformations of cellulose modification.

solution for $1 \mathrm{~h}$, which was followed by thorough rinsing with deionized water to remove all residual reagents and byproducts.

\section{Characterization of solute rejection}

The cellulose membranes were highly hydrophilic porous materials. That excluded any characterization of the degree of membrane modification by a gravimetric method. Indirect indications of the modification process were based on changes in transport properties, namely, the pure water transmembrane flux and solute rejection. Characterization of the initial membranes demonstrated excellent reproducibility of the water flux and solute rejection within a separate membrane sheet and between different sheets. This allowed estimation of the modification degree because changes in the water transmembrane flux and solute rejection correlated with the quantity of chitosan bound to the membrane surface. The pure water transmembrane flux and solute rejection were determined in an Amicom 8200 cylindrical dead-end filtration cell (Millipore) at room temperature. The operating pressure was varied between 50 and $200 \mathrm{kPa}$. Solute rejection was determined with model solutions of a single solute. $\mathrm{NaCl}, \mathrm{MgCl}_{2}$, and PEGs with molecular weights of 4600 and 6000 were used to determine the rejection properties of the prepared membranes. For the filtration experiments, solutions were used with a concentration of $1 \mathrm{mM}$ for electrolytes and $1 \mathrm{~g} / \mathrm{L}$ for PEG solutions. The water flux and rejection were determined as average values from three samples of modified and unmodified membranes.

Conductivity measurements were used to determine electrolyte concentrations in feed and permeate solutions. The concentration of PEG in feed and per- meate solutions was determined with a differential refractometer. The solute rejection $(R)$ was calculated with the following formula:

$$
R=\left(1-\frac{C_{p}}{C_{f}}\right) \cdot 100 \%
$$

where $C_{f}$ and $C_{p}$ are the solute concentrations in the feed and permeate, respectively.

\section{Attenuated total reflectance/infrared (ATR-IR) spectroscopy}

A Bruker IFS-55 Fourier transform infrared spectrometer (Bruker Optics, Ltd., Ontario, Canada) was used to analyze the chemical modifications produced in the approximately outermost $0.5-1 \mu \mathrm{m}$ of the treated membrane surfaces. A Spectra-Tech Baseline ATR attachment (Shelton, CT) equipped with a minigrip pressure device and a germanium ATR prism was used. The IR beam entered the ATR-IR device at an angle of $45^{\circ}$, and 100 scans were accumulated with a resolution of $4 \mathrm{~cm}^{-1}$.

\section{Evaluation of the membrane antimicrobial activity}

Gram-negative E. coli BE and gram-positive S. aureus CCM 209 bacteria strains were received from the Ukrainian Collection of Microorganisms. The bacteria were grown and maintained in Nutrient Agar No. 1 (Fluka).

Microbial suspensions were prepared in a sterilized physiological solution. Fifty-microliter suspensions containing $(4-5) \times 10^{5}$ cells $/ \mathrm{mL}$ were diluted in $50 \mathrm{~mL}$ of a physiological solution and then were filtered through the membrane at an operating pressure of $200 \mathrm{kPa}$. After filtration, the membranes 
were incubated in Nutrient Agar medium (S. aureus) or Endo Agar medium (E. coli) for $24 \mathrm{~h}$ at $30^{\circ} \mathrm{C}$. The bactericidal activity was determined in terms of the growth inhibition, which was calculated with the following equation:

$$
\text { Growth inhibition }=\frac{N_{1}-N_{2}}{N_{1}} \times 100 \%
$$

where $N_{1}$ and $N_{2}$ are the numbers of viable colonies on control and modified membranes, respectively. An unmodified membrane was used as a control.

\section{RESULTS AND DISCUSSION}

\section{Chemical modification of the cellulose membrane surface}

According to the scheme of cellulose modification, chitosan tethering to the membrane surface is based on cellulose activation via the introduction of aldehyde groups into the molecular structure of cellulose. Aldehyde groups can be detected by IR analysis as a band at $1740-1720 \mathrm{~cm}^{-1}$, which is characteristic of carbonyl group stretching. As can be seen in Figure 1, this characteristic band appears after cellulose oxidation with periodate. Often, the presence of absorbed or bound water molecules makes it difficult to identify aldehyde groups. Bending vibrations of water molecules give a characteristic band at about $1640 \mathrm{~cm}^{-1}$ that can be very broad. This can hide the bands of carbonyl groups. Figure 1 demonstrates that the band characteristic of water molecules can be observed for both the cellulose membrane and the cellulose membrane after its oxidation. However, for the oxidized membrane, the bands of water molecules $\left(1643 \mathrm{~cm}^{-1}\right)$ and carbonyl groups $\left(1737 \mathrm{~cm}^{-1}\right)$ are clearly distinguishable. This indicates the formation of dialdehyde cellulose after its modification with sodium periodate.

Chitosan has a series of bands between 1750 and $1500 \mathrm{~cm}^{-1}$. The band at $1648 \mathrm{~cm}^{-1}$ is ascribed to stretching of the carbonyl group of the amide I type, which is characteristic for $\mathrm{N}$-acetylated chitin. However, water molecules interfere with this band. A band at $1574 \mathrm{~cm}^{-1}$ shows an $\mathrm{NH}_{2}$-associated bond. Thus, a spectral area between 1750 and $1500 \mathrm{~cm}^{-1}$ contains characteristic bands of carbonyl and amine groups and water molecules. This complicates the identification of the contribution of a specific functional group to the spectra of cellulose membranes after chitosan binding. However, the treatment of an oxidized cellulose membrane with chitosan results in a change in the ratio of band intensities at 1740 and $1640 \mathrm{~cm}^{-1}$. For a cellulose membrane with tethered chitosan, the peak at $1740 \mathrm{~cm}^{-1}$ becomes more intense than the peak at $1640 \mathrm{~cm}^{-1}$. Moreover, a broad band around $3300 \mathrm{~cm}^{-1}$, which corresponds

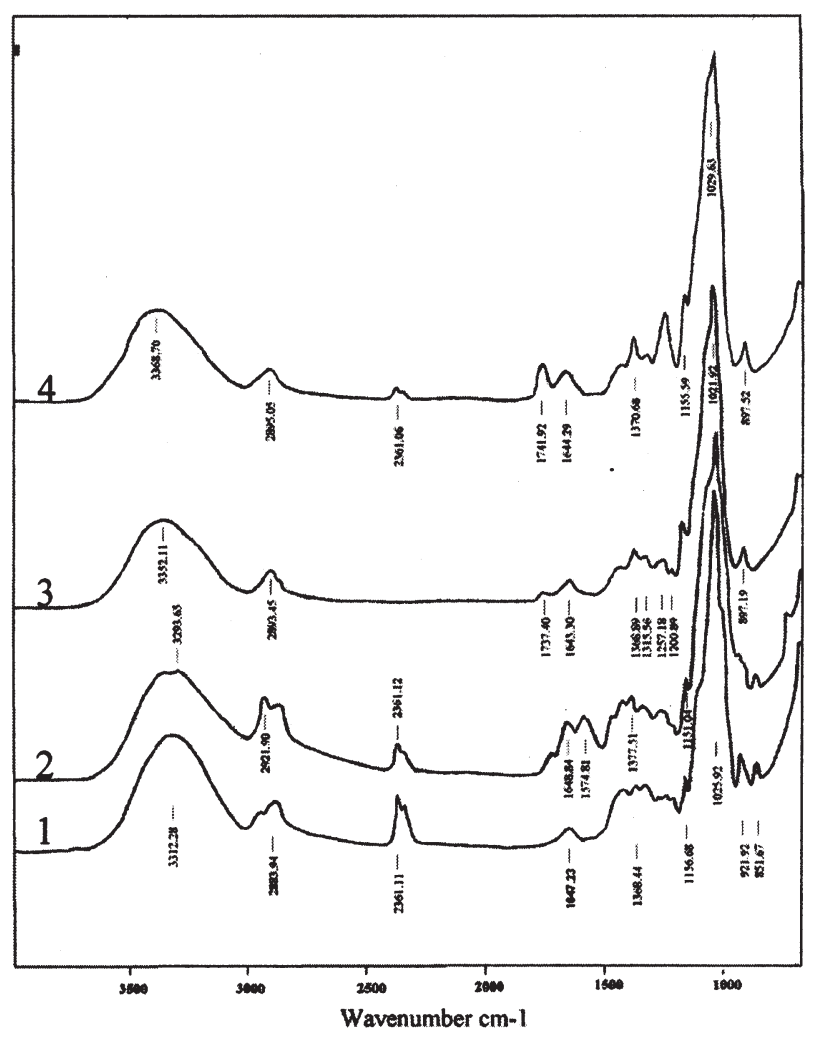

Figure 1 ATR-IR spectra of (1) a cellulose membrane, (2) a chitosan film, (3) a membrane after periodate oxidation, and (4) an oxidized membrane after treatment with a chitosan solution.

to $\mathrm{O}-\mathrm{H}$ and $\mathrm{N}-\mathrm{H}$ stretching, shifts toward high frequencies after the treatment of oxidized membranes with chitosan. These changes in the peak intensities and positions between 1740 and $1640 \mathrm{~cm}^{-1}$ and around $3300 \mathrm{~cm}^{-1}$ might be attributed to the binding of chitosan to aldehyde groups of oxidized cellulose.

Further confirmation of chitosan binding can be proven from both membrane surface properties and transmembrane transport. Generally, membrane modification from the solution complicates nondestructive determinations of the degree of modification. Changes in solvent convective flow for porous membranes give a good indication of the alteration of the porous structure. Thus, variations in the degree of membrane modification can be indirectly characterized in terms of transmembrane flux.

An oxidation step using the chosen conditions does not affect the water transmembrane flux or rejection of PEGs. However, as can be seen in Figure 2 , the treatment of oxidized membranes with chitosan solutions results in a decrease in the water flux, which indicates the binding of chitosan molecules to the activated cellulose membranes. The hydraulic permeability of modified membranes gradually decreases with the growth of the chitosan concentration up to $10 \mathrm{~g} / \mathrm{L}$. A further increase in the chitosan 


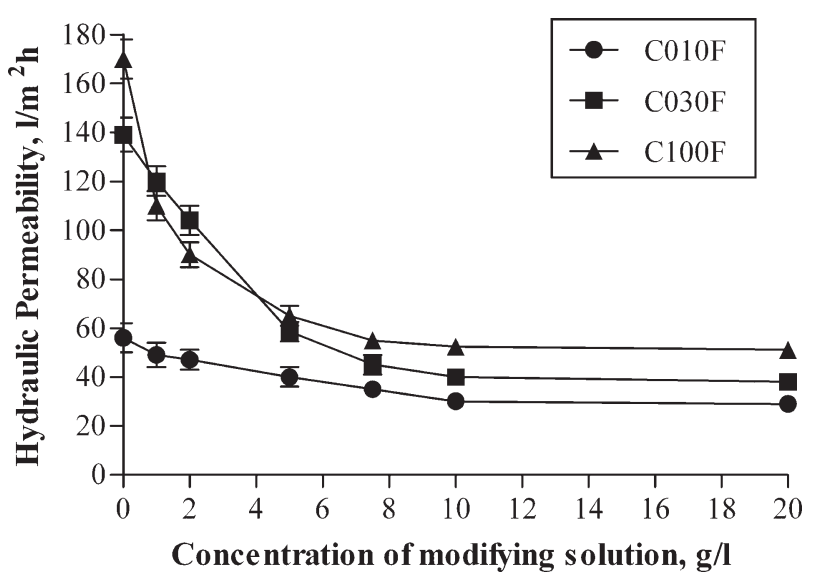

Figure 2 Effect of the chitosan concentration in treatment solutions on the hydraulic permeability of membranes. The membranes were modified by chitosan with a molecular weight of $400 \mathrm{kDa}$ at $\mathrm{pH}=3.8$.

concentration does not lead to an increase in the degree of modification. Chitosan macromolecules can be tethered to the surface of an activated membrane via one point or several points. The latter happens if several amine groups of the same macromolecule react with aldehyde groups of activated cellulose. Multipoint binding decreases the number of chitosan macromolecules that can be bound to the membrane surface and consequently restricts the degree of membrane modification. At a low chitosan concentration, multipoint binding prevails, and the degree of membrane modification is low. An appreciable decrease in the membrane hydraulic permeability can be observed when the concentration of chitosan exceeds $3 \mathrm{~g} / \mathrm{L}$. As the chitosan concentration grows, the competitiveness of the macromolecules increases. This results in predominantly one-point binding at chitosan concentrations higher than $10 \mathrm{~g} / \mathrm{L}$. As a result, the degree of membrane modification approaches a maximal value and does not change with chitosan concentrations higher than $10 \mathrm{~g} / \mathrm{L}$.

A similar pattern of the influence of the chitosan concentration on membrane hydraulic permeability can be observed for cellulose membrane modification with chitosans of two molecular weights, 400 and $150 \mathrm{kDa}$. However, with predominantly onepoint binding, the degree of modification is expected to be higher in the case of large molecules. As Figure 3 shows, for the same modification conditions, the higher molecular weight chitosan causes a larger decrease in membrane hydraulic permeability than the lower molecular weight chitosan for membranes with smaller pore sizes, C005F and C010F. Because the molecular weight of the chitosan is significantly higher than the nominal MWCO of these membranes, chitosan molecules do not penetrate mem- brane pores, so the chitosan layer is formed entirely on the membrane surface. Thus, the lower hydraulic permeability of $\mathrm{C} 005 \mathrm{~F}$ and $\mathrm{C} 010 \mathrm{~F}$ membranes modified with the higher molecular weight chitosan is due to the formation of thicker layers of immobilized chitosan on the membrane surface. Such layers have higher hydraulic resistance, which causes a more prominent decrease in the transmembrane convective flow. However, a slight increase in the rejection of PEG 4000 was observed for C005F and C010F membranes modified with the lower molecular weight chitosan versus the same membranes that were modified by the higher molecular weight chitosan (Table I). It is known that the flexibility of chitosan molecules increases with the growth of the molecular weight. ${ }^{26}$ This could result in the formation of denser chitosan layer of less flexible chitosan with a molecular weight of $150 \mathrm{kDa}$. With a high density of aldehyde groups on the surface of membranes with smaller pore sizes, the probability of multiple binding increases, and this leads to more compact binding of less flexible chitosan molecules.

When the membrane pore size is comparable to the molecular size of the chitosan molecules, chitosan binding can occur inside membrane pores. As shown in Figure 3, for membranes with larger pore sizes, C030F and C100F, the lower molecular weight chitosan reduces membrane permeability either to the same extent or even more than the higher molecular weight chitosan. The lower molecular weight chitosan causes a partial blockage of the largest pores. Thus, the $\mathrm{C} 100 \mathrm{~F}$ membrane with the largest pore size has lower hydraulic permeability after modification with the lower molecular weight chitosan than when it is modified with the higher molecular weight chitosan. Macromolecules of the 400$\mathrm{kDa}$ chitosan cannot be accommodated by pores of either membrane, and only surface modification is achievable with this chitosan. Surprisingly, the

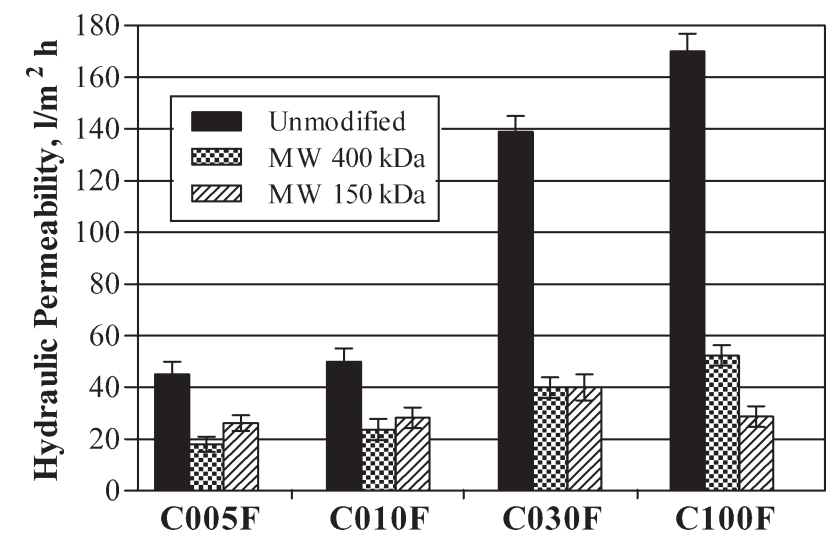

Figure 3 Influence of the molecular weight of chitosan on the hydraulic permeability of cellulose membranes with different pore sizes. 
TABLE I

Separation Performance of Membranes Modified by Chitosans of Different Molecular Weights

\begin{tabular}{|c|c|c|c|c|c|c|}
\hline \multirow[b]{2}{*}{ Membrane } & \multicolumn{2}{|c|}{ Initial membrane } & \multicolumn{2}{|c|}{$\begin{array}{l}\text { Modification with 400-kDa } \\
\text { chitosan }\end{array}$} & \multicolumn{2}{|c|}{$\begin{array}{l}\text { Modification with } 150-\mathrm{kDa} \\
\text { chitosan }\end{array}$} \\
\hline & $R_{\text {PEG }}(\%)^{\mathrm{a}}$ & $R(\%)^{\mathrm{d}}$ & $R_{\mathrm{PEG}}(\%)^{\mathrm{a}}$ & $\overline{R(\%)^{\mathrm{d}}}$ & $R_{\mathrm{PEG}}(\%)^{\mathrm{a}}$ & $R(\%)^{\mathrm{d}}$ \\
\hline C005F & $85.4 \pm 0.6^{\mathrm{b}}$ & $\frac{3.8 \pm 0.4}{6.5 \pm 0.8}$ & $98.8 \pm 0.5^{\mathrm{b}}$ & $\frac{14.6 \pm 0.8}{29.6 \pm 0.5}$ & $99.5 \pm 0.3^{\mathrm{b}}$ & $\frac{8.5 \pm 0.6}{22.5 \pm 0.4}$ \\
\hline C010F & $78.9 \pm 0.6^{\mathrm{b}}$ & $\frac{3.1 \pm 0.6}{6.3 \pm 0.5}$ & $84.5 \pm 0.6^{\mathrm{b}}$ & $\frac{15.0 \pm 0.5}{30.7 \pm 0.5}$ & $90.8 \pm 0.4^{\mathrm{b}}$ & $\frac{5.2 \pm 0.5}{13.5 \pm 0.8}$ \\
\hline $\mathrm{CO30F}$ & $61.2 \pm 0.6^{\mathrm{C}}$ & $\frac{0}{0}$ & $98.8 \pm 0.3^{c}$ & $\frac{6.5 \pm 0.6}{7.9 \pm 0.5}$ & $97.0 \pm 0.5^{c}$ & $\frac{0.6 \pm 0.5}{4.6 \pm 0.3}$ \\
\hline $\mathrm{C} 100 \mathrm{~F}$ & $4.2 \pm 0.8^{\mathrm{c}}$ & $\frac{0}{0}$ & $94.6 \pm 0.5^{c}$ & $\frac{0}{0}$ & $92.9 \pm 0.6^{\mathrm{c}}$ & $\frac{0}{0}$ \\
\hline
\end{tabular}

a Rejection of PEG.

b Rejection of PEG 4000 (operating pressure $=0.2 \mathrm{MPa}$ ).

${ }^{\mathrm{c}}$ Rejection of PEG 20,000 (operating pressure $=0.05 \mathrm{MPa}$ ).

${ }^{\mathrm{d}}$ Rejection of inorganic electrolytes: $\mathrm{NaCl}$ (upper figures) and $\mathrm{MgCl}_{2}$ (lower figures). The operating pressure was $0.2 \mathrm{MPa}$ for membranes C005F and C010F and 0.05 MPa for membranes C030F and C100F.

rejection of PEG is lower in the case of $\mathrm{C} 030 \mathrm{~F}$ and C100F membranes modified with the 150-kDa chitosan. For these membranes, the surface density of aldehyde groups is lower, and this could result in nonuniform coverage of the membrane surface with more rigid chitosan of a smaller molecular size.

As can be seen in Table II, tethering chitosan to the membrane surface results in a change in the surface charge from negative to positive. Membranes modified with chitosan of a higher molecular weight have a consistently higher positive charge than membranes modified with chitosan with a molecular weight of $150 \mathrm{kDa}$. This confirms the assumption that chitosan with a molecular weight of $400 \mathrm{kDa}$ provides a higher degree of modification. The surface charge of the modified membranes decreases with the increase in the pore size of the initial membranes. As mentioned previously, the surface density of aldehyde groups decreases when the membrane pore size increases. Therefore, the overall degree of modification and consequently surface charge decrease with the increase in the membrane pore size.

Surface-charged porous membranes are capable of rejecting inorganic electrolytes at low concentrations if the pore sizes are sufficiently small. ${ }^{27}$ As Table I shows, modified C005F and C010F membranes show some retention of $\mathrm{NaCl}$ and $\mathrm{MgCl}_{2}$. Significantly higher retention of the $2: 1$-type electrolyte versus the 1 : 1-type electrolyte is concordant with an electrochemical mechanism of electrolyte rejection by porous charged membranes. Membranes modified with the higher molecular weight chitosan have higher charge density (Table II) and consequently higher electrolyte rejection.

Thus, the physicochemical and transport characteristics of the prepared membranes confirm the modification of cellulose membranes under the chosen conditions according to the scheme of chemical transformations (Scheme 1). With the use of chito- sans with different molecular weights and membranes with different pore sizes, the alteration of the morphological structure of tethered chitosan layers is achievable.

\section{Antibacterial activity of the cellulose membranes modified with chitosan}

All membranes involved in this study provide complete retention of bacterial cells. The filtration of a specific volume of a bacterial suspension allows the quantitative transformation of bacteria onto the membrane surface as cells precipitate. Figure 4 shows representative pictures of such cell-fouled membrane samples after their incubation on nutrient media. Membranes modified by chitosan demonstrate a drastic decrease in the number of viable cells versus unmodified membranes. As can be seen in Figure 5, the antimicrobial activity of modified membranes depends on the degree of membrane modification. For example, in the case of the C010F membrane, there is substantial antibacterial activity when the chitosan concentration in the reaction medium is higher than $5 \mathrm{~g} / \mathrm{L}$, that is, when the degree of membrane modification approaches its maximal value (see Fig. 1). However, bactericidal activity

TABLE II

$\zeta$-Potential of the Initial Cellulose Membranes and the Membranes Modified with Chitosans of Different Molecular Weights

\begin{tabular}{cccc}
\hline & \multicolumn{3}{c}{$\zeta$-Potential $(\mathrm{mV})$} \\
\cline { 2 - 4 } & Initial & $\begin{array}{c}\text { Membrane } \\
\text { modified } \\
\text { with 150-kDa } \\
\text { chitosan }\end{array}$ & $\begin{array}{c}\text { Membrane } \\
\text { modified } \\
\text { with 400-kDa } \\
\text { chitosan }\end{array}$ \\
\hline C005F & $-14.89 \pm 1.12$ & $+7.28 \pm 0.39$ & $+9.44 \pm 0.60$ \\
C030F & $-13.11 \pm 1.52$ & $+6.40 \pm 0.37$ & $+7.80 \pm 0.51$ \\
C100F & $-15.77 \pm 1.02$ & $+2.46 \pm 1.43$ & $+3.64 \pm 1.05$ \\
\hline
\end{tabular}




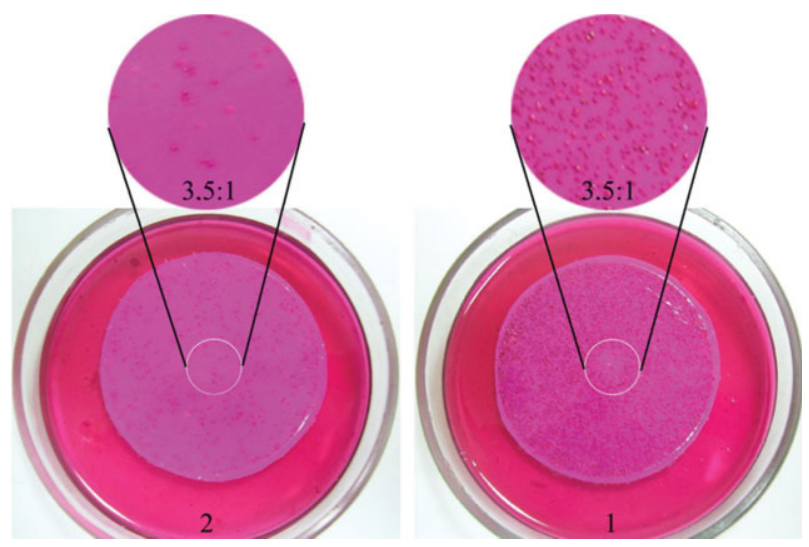

Figure 4 Microbial growth on the surface of (1) the C010F membrane and (2) the C010F membrane modified with chitosan with a molecular weight of $400 \mathrm{kDa}$. [Color figure can be viewed in the online issue, which is available at www.interscience.wiley.com.]

reaches $99 \%$ after modification using a chitosan solution with a concentration of $7.5 \mathrm{~g} / \mathrm{L}$. With such a modification condition, the degree of modification does not reach its maximal value. Nevertheless, the membrane surface provides virtually complete killing of the deposited bacterial cells.

Although it is widely accepted that the antimicrobial activity of cationic polymers including chitosan is not fully understood, it is believed that the binding of positively charged polycations to the surface of negatively charged cells and the disruption of at least outer cells of the membrane are involved in the processes affecting cell viability. ${ }^{25,28}$ According to these postulations, an increase in the surface charge is beneficial for the antimicrobial activity of membranes because of improved efficiency of bacterial cell binding by positively charged membranes. Therefore, the antimicrobial activity of membranes

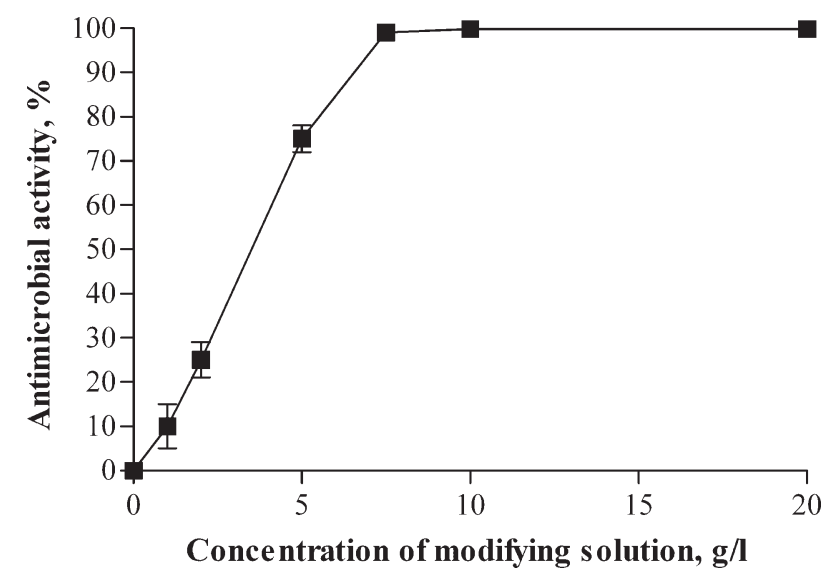

Figure 5 Effect of the chitosan concentration in treatment solutions on the antimicrobial activity of modified membranes. Cellulose membrane C010F was modified by chitosan with a molecular weight of $400 \mathrm{kDa}$ at $\mathrm{pH}=3.8$. modified with chitosan increases with the growth of the degree of membrane modification.

Another factor crucial to the antimicrobial activity is the ability of polycationic chains or chain segments to disrupt the cellular barrier. This ability is influenced by many properties of polymeric biocides, such as the molecular weight, chain flexibility, and hydrophilic/hydrophobic balance. Moreover, in the case of biocides immobilized on a solid surface, the supramolecular and morphological structures of the composite material are expected to be important for antimicrobial activity. According to Figure 6, the efficiency of antimicrobial action against grampositive S. aureus and gram-negative E. coli for cellulose membranes modified with chitosan is dependent on the porous structure of the membrane and molecular weight of chitosan. Membranes with tethered chitosan are more active against gram-positive bacteria. An increase in the membrane pore size tends to cause a decline in the membrane antimicrobial efficiency. However, all the studied membranes modified with higher molecular weight chitosan showed activity against $S$. aureus of no less than $95 \%$, but membrane modification with 150-kDa chitosan provided high antimicrobial activity only for

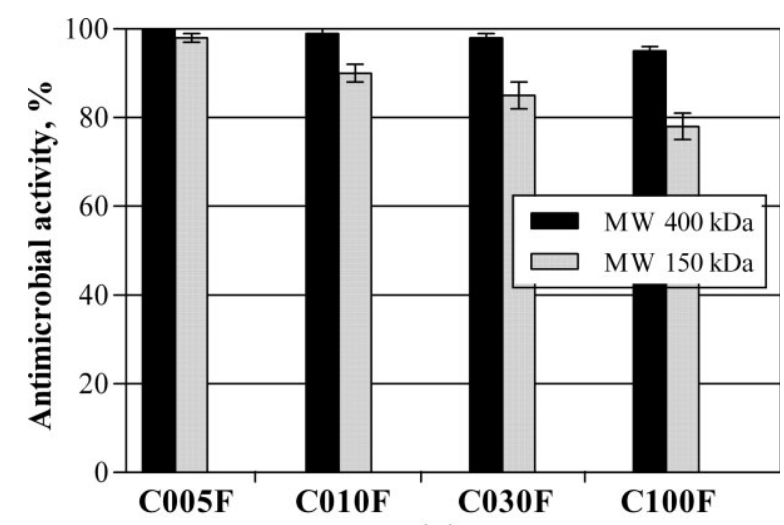

(a)

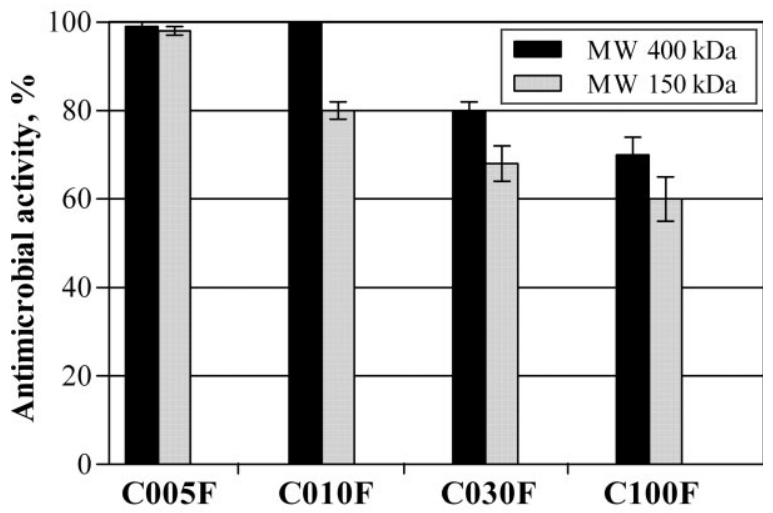

(b)

Figure 6 Effects of the molecular weight of chitosan and the membrane pore size on antimicrobial activity against (a) S. aureus and (b) E. coli. 
C005F membrane, membrane with the smallest pore size. The efficiency of membranes with larger pore sizes against $S$. aureus are notably lower when lower molecular weight chitosan is used as a modifying agent.

Similarly, the antimicrobial activity against gramnegative E. coli is higher for membranes modified with higher molecular weight chitosan. However, even in the case of higher molecular weight chitosan, activity against E. coli higher than $95 \%$ was reached only for membranes $\mathrm{C} 005 \mathrm{~F}$ and $\mathrm{C} 010 \mathrm{~F}$. Lower molecular weight chitosan afforded such activity only in the case of the modification of the membrane with the smallest pore size, C005F.

The results regarding the influence of the molecular weight of chitosan on the antimicrobial activity are concordant with findings published by other groups using polymeric biocides bound to solid surfaces. ${ }^{29,30}$ Textile materials modified with chitosan ${ }^{29}$ and alkylated polyethylenimine ${ }^{30}$ are more efficient against both gram-positive and gram-negative bacteria with an increase in the molecular weight of the polymeric biocide. It has been postulated that immobilized biocides must be sufficiently long to traverse and damage the cellular membrane/wall. ${ }^{30}$ From this point of view, a porous material can significantly alter the antimicrobial activity because polymer protrusion from the surface is variable on account of surface/polymer interactions, the possibility of the accommodation of the polymer (or at least part of the molecule) within the porous structure, and so forth. As discussed in the previous section, the modification of membranes with smaller pore sizes $(\mathrm{C} 005 \mathrm{~F}$ and $\mathrm{C} 010 \mathrm{~F})$ occurs entirely on the membrane surface when the membranes are modified with higher molecular weight chitosan. This results in the most expanded layer of bound chitosan with the highest activity against both $S$. aureus and E. coli. With an increase in the membrane pore size, polymer segments can penetrate membrane pores. This decreases the effective length of chitosan chains protruding from the membrane surface, especially in the case of lower molecular weight chitosan. As a result, the antimicrobial efficiency gradually decreases when the pore size of the cellulose membrane increases. This decrease in the antimicrobial activity is more pronounced for membranes modified with lower molecular weight chitosan. It is worth noting that the penetration of segments of polymer chains into membrane pores increases the probability of multipoint binding. This also restricts the conformational freedom of tethered polymer chains and subsequently the effective length of polymer chains protruding from the surface. Therefore, one can suggest that this factor also contributes to the reduction of the antimicrobial activity of modified membranes with a larger pore size.
Soluble forms of chitosan usually show higher antimicrobial efficiency against gram-positive bacteria. ${ }^{31}$ This pattern of antimicrobial activity for immobilized chitosan remains at least for the two studied bacterial strains, S. aureus and E. coli; membranes modified with chitosans of both molecular weights are consistently more active against $S$. aureus than E. coli. The higher activity of cationic chitosan against gram-positive bacteria is mainly attributable to the higher surface charge of these bacteria versus gram-negative bacteria. ${ }^{31}$ Thus, chitosan provides a stronger attachment to the surface of gram-positive bacteria. Such intimate contact improves the efficiency of polymer molecules in the distortion of cell walls. As shown in the previous section, higher molecular weight chitosan renders the membrane surface more positively charged than lower molecular weight chitosan. Therefore, higher antimicrobial activity against gram-positive $S$. aureus for membranes with tethered higher molecular weight chitosan can also be explained by more efficient binding of the bacteria to the membrane surface.

\section{CONCLUSIONS}

In an effort to render the surface of semipermeable membranes biocidal and thereby to lower membrane biofouling potential, cellulose membranes were modified with chitosan, a naturally occurring polycationic biocide. Cellulose activation was implemented via oxidation with the introduction of aldehyde groups. The latter were used to attach chitosan molecules to the membrane surface via primary amino groups. Cellulose membranes of different pore sizes were modified with higher molecular weight chitosan $(400 \mathrm{kDa})$ and lower molecular weight chitosan $(150 \mathrm{kDa})$.

Higher molecular weight chitosan provides a higher degree of modification, which results in a higher positive charge of the membrane surface versus modification using lower molecular weight chitosan. From the transport and surface characteristics of prepared membranes, it has been concluded that for membranes with larger pore sizes, at least part of the chitosan molecule can be located inside the porous structure. This trend is more pronounced for lower molecular weight chitosan. On the contrary, the binding of higher molecular weight chitosan occurs entirely on the surface for membranes with the smallest pore size. Thus, with the use of chitosans of different molecular weights and membranes of different pore sizes, an alteration of the morphological structure of tethered chitosan layers can be achieved.

The importance of such structural differences for antimicrobial activity of the prepared membranes has been studied with the use of gram-positive 
S. aureus and gram-negative E. coli. The highest antimicrobial activity of modified membranes is achievable when the degree of membrane modification is close to its maximal value. Antimicrobial efficiency improves with the use of higher molecular weight chitosan and membranes with smaller pore sizes. Such dependences are postulated to be associated with the effective length of chitosan chains protruding from the membrane surface. It is believed that immobilized biocides must be sufficiently long to traverse and damage the cellular membrane/wall of bacterial cells in contact with the surface. Modification of membranes with smaller pore sizes occurs entirely on the membrane surface when membranes are modified with higher molecular weight chitosan. This results in the most expanded layer of bound chitosan with the highest activity against both $S$. aureus and E. coli. The effective length of chitosan chains projecting from the membrane surface decreases with the growth of the membrane pore size because of polymer segment penetration into membrane pores. This is more important for lower molecular weight chitosan. Consequently, the decrease in the antimicrobial efficiency with the increase in the pore size is more pronounced when membranes are modified with lower molecular weight chitosan.

Membranes modified with chitosans of both molecular weights are consistently more active against gram-positive $S$. aureus than gram-negative E. coli. Generally, gram-positive bacteria are characterized by a higher negative surface charge. Membranes with tethered higher molecular weight chitosan probably provide more efficient binding of $S$. aureus because of the higher positive charge of their surface and consequently higher antimicrobial activity.

\section{References}

1. Al-Ahmad, M.; Aleem, F. A. A.; Mutiri, A.; Ubaisy, A. Desalination 2000, 132, 173.

2. Baker, J. S.; Dudley, L. Y. Desalination 1998, 118, 81.
3. Flemming, H. C. Appl Microbiol Biotechnol 2002, 59, 629.

4. Flemming, H. C.; Schaule, G.; Griebe, T.; Schmitt, J.; Tamachkiarowa, A. Desalination 1997, 113, 215.

5. Park, N.; Kwon, B.; Kim, S.; Cho, J. J Membr Sci 2005, 258, 43.

6. Saad, M. A. Desalination 1992, 88, 85.

7. Saeed, M. O.; Jamaluddin, A. T.; Tisan, I. A.; Lawrence, D. A.; Al-Amri, M. M.; Chida, K. Desalination 2000, 128, 177.

8. Speth, T. F.; Summers, R. S.; Gusses, A. M. Environ Sci Technol 1998, 32, 3612.

9. Schneider, R. P.; Ferreira, L. M.; Binder, P.; Ramos, J. R. J Membr Sci 2005, 261, 152.

10. Ridgway, H. F.; Kelly, A.; Justice, C.; Olson, B. H. Appl Environ Microbiol 1983, 45, 1066.

11. Ross, N.; Deschenes, L.; Bureau, J.; Clement, B.; Comeau, Y.; Samson, R. Environ Sci Technol 1998, 32, 1105.

12. Kaeselev, B.; Pieracci, J.; Belfort, G. J Membr Sci 2001, 194, 245.

13. Hamza, A.; Pham, V. A.; Matsuura, T.; Santerre, J. P. J Membr Sci 1997, 131, 217.

14. Carroll, T.; Booker, N. A.; Meier-Haack, J. J Membr Sci 2002, 203, 3 .

15. Bowen, W. R.; Doneva, T. A.; Yin, H. B. J Membr Sci 2002, 206, 417.

16. Danese, P. N. Chem Biol 2002, 9, 873.

17. Stashak, T. S.; Farstvedt, E.; Othic, A. Clin Technol Equine Pract 2004, 3, 148.

18. Imazato, S.; Ebi, N.; Takahashi, Y.; Kaneko, T.; Ebisu, S.; Russell, R. R. B. Biomaterials 2003, 24, 3605.

19. Bajaj, P. J Appl Polym Sci 2002, 83, 631.

20. Appendini, P.; Hotchkiss, J. H. Innov Food Sci Emerg Technol 2002, 3, 113.

21. Tiller, J. C.; Lee, S. B.; Lewis, K.; Klibanov, A. M. Biotechnol Bioeng 2002, 79, 465.

22. Lea, S. B.; Koepsel, R. R.; Morley, S. W.; Matyjaszewski, K.; Sun, Y.; Russel, A. J. Biomacromolecules 2004, 5, 877.

23. Hilal, N.; Kochkodan, V.; Al-Khatib, L.; Levadna, T. Desalination 2004, 167, 293.

24. Hilal, N.; Al-Khatib, L.; Atkin, B. P.; Kochkodan, V.; Potapchenko, N. Desalination 2003, 158, 65.

25. Rabea, E. I.; Badawy, M. E.-T.; Stevens, C. V.; Smagghe, G.; Steurbaut, W. Biomacromolecules 2003, 4, 1457.

26. Chen, R. H.; Tsaih, M. L. Int J Biol Macromol 1998, 23, 135.

27. Yaroshchuk, A. E. Adv Colloid Interface Sci 1995, 60, 1.

28. Helander, I. M.; Nurmiaho-Lassila, E.-L.; Ahvenainen, R.; Rhoades, J.; Roller, S. Int J Food Microbiol 2001, 71, 235.

29. Shin, Y.; Yoo, D. I.; Jang, J. J Appl Polym Sci 2001, 80, 2495.

30. Lin, J.; Qiu, S.; Lewis, K.; Klibanov, A. M. Biotechnol Bioeng 2003, 83, 168 .

31. Chung, Y. C.; Su, Y. P.; Chen, C. C.; Jia, G.; Wang, H. L.; Wu, J. C. G.; Lin, J. G. Acta Pharmacol Sin 2004, 25, 932. 